\title{
A Case Report: Puerperal Ovarian Cancer in a Patient with a Prior History of Fertility Treatment and Ovarian Endometrial Cyst
}

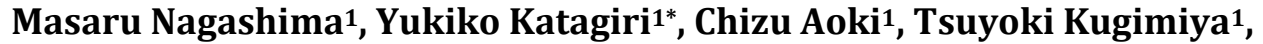 \\ Yoshihiro Yoshida', Michiko Saigusa', Kazuo Masaki'1, Kazutoshi Shibuya'2, Mineto Morita1 \\ ${ }^{1}$ Department of Obstetrics and Gynecology, Toho University, Omori Medical Center, Tokyo, Japan \\ ${ }^{2}$ Department of Pathology, Toho University, Omori Medical Center, Tokyo, Japan \\ Email: ‘yukikonk@med.toho-u.ac.jp
}

Received 2 May 2014; revised 1 June 2014; accepted 8 June 2014

Copyright (C) 2014 by authors and Scientific Research Publishing Inc.

This work is licensed under the Creative Commons Attribution International License (CC BY).

http://creativecommons.org/licenses/by/4.0/

c) (i) Open Access

\begin{abstract}
Introduction: It has been suggested the relationship between infertility treatment and ovarian cancer. Furthermore, endometriosis that is one of the causes of the infertility tends to develop into endometrioid adenocarcinoma and clear cell carcinoma in many studies. Case: A 38-year-old woman underwent laparoscopic enucleation of bilateral ovarian endometrial cysts at 29 and 33 years old. She then received 11 sessions of artificial insemination and 7 cycles of ovarian stimulation, egg collection, in vitro fertilization, and embryo transfer at multiple medical institutions before conceiving and giving birth to her first child. After naturally conceiving her second child, she presented at our hospital for pregnancy/delivery management with a right ovarian tumor. She underwent abdominal right adnexectomy for a mucinous cystic tumor of borderline malignancy at 36 years old (17 weeks of pregnancy with the second child). After vaginal delivery at 38 weeks, a 1-month postpartum checkup revealed a left adnexal mass and ascites. Further workup led to a diagnosis of left ovarian cancer. The cancer was diagnosed by ovarian biopsy as unresectable stage-IIIc endometrioid carcinoma. Despite chemotherapy, the patient developed liver metastasis during treatment and died due to worsened general condition 9 months after childbirth. In addition to ovarian endometrial cyst, repeated cycles of injury and repair of the ovarian surface epithelium are also suggested to mediate the development of epithelial ovarian cancer. Conclusion: Evaluation of long-term outcome and follow-up of patients who have undergone fertility treatment should be addressed.
\end{abstract}

\section{Keywords}

Puerperal, Ovarian Cancer, Sterility, Assisted Reproductive Technology, Endometriosis

\footnotetext{
"Corresponding author.
}

How to cite this paper: Nagashima, M., Katagiri, Y., Aoki, C., Kugimiya, T., Yoshida, Y., Saigusa, M., Masaki, K., Shibuya, K. and Morita, M. (2014) A Case Report: Puerperal Ovarian Cancer in a Patient with a Prior History of Fertility Treatment and Ovarian Endometrial Cyst. Open Journal of Obstetrics and Gynecology, 4, 550-556. 


\section{Introduction}

Although some studies have suggested a correlation between fertility treatments based mainly on the use of ovulation-inducing agents and the development of malignancy, more studies have suggested that ovulation induction treatment does not increase the short-term risk of ovarian cancer [1] [2]. Meanwhile, other studies have suggested that infertility itself can be a risk factor for ovarian cancer. Many recent studies have also shown that an ovarian endometrial cyst is prone to develop into endometrioid adenocarcinoma and clear cell carcinoma [3] [4]. This report describes a case of ovarian cancer during puerperium in a patient with endometriosis and a prior history of long-term fertility treatment.

\section{Case}

Patient: a 38-year-old woman who had been pregnant twice and given birth to 2 children.

Chief complaint: a left adnexal mass.

Menstrual history: a 35-day regular menstrual cycle; irregular and oligomenorrhic before conceiving her first child.

Catamenial disorders: pain/anal pain during menstruation and menorrhagia.

Past medical history: laparoscopic enucleation of bilateral ovarian endometrial cysts at 29 and 33 years old, abdominal right adnexectomy at 36 years old (17 weeks of pregnancy with second child).

History of present illness: since getting married at the age of 24, she tried to become pregnant, but despite no contraception she was unsuccessful. She started fertility treatment at the age of 27, continued the treatment for 8 years, and finally conceived her first child at the age of 34. Following 11 sessions of artificial insemination, she underwent a total of 17 cycles of egg collection at 3 medical institutions. Although no findings suggestive of endometriosis were observed at the beginning of fertility treatment, bilateral ovarian endometrial cysts were subsequently detected, and 2 sessions of laparoscopic bilateral cystectomy were performed at 29 and 33 years old (Figure 1). The history of fertility and endometriosis treatment and the list of drugs used for the ovarian stimulation during fertility treatment are summarized in Table 1. When the patient was found to be pregnant with
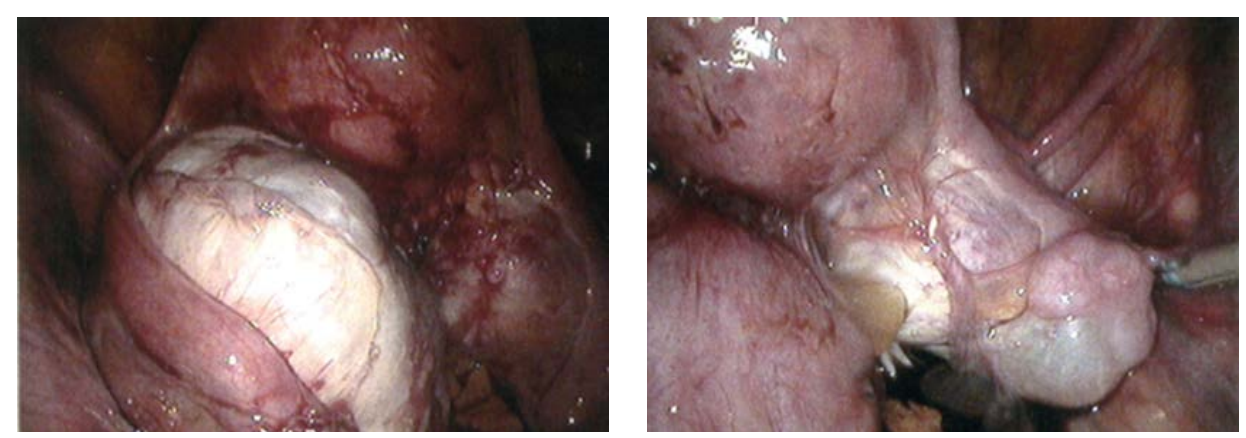

Figure 1. Laparoscopic enucleation of bilateral ovarian endometrial cysts. The diameters were 3 $\mathrm{cm}$ on the right side and $6 \mathrm{~cm}$ on the left side. Pathological diagnosis: endometrial cyst.

Table 1. Names and total dose of drugs used for ovulation induction during fertility treatment.

\begin{tabular}{cc}
\hline Generic name & Total dose \\
\hline Clomiphene hydrochloride & $4350 \mathrm{mg}$ \\
Cyclofenil & $2000 \mathrm{mg}$ \\
Letrozole & $25 \mathrm{mg}$ \\
uhMG & $5850 \mathrm{IU}$ \\
uFSH & $450 \mathrm{IU}$ \\
recFSH & $1050 \mathrm{IU}$ \\
\hline
\end{tabular}


her second child at the age of 37, a 7-cm right ovarian tumor was noted, and abdominal right adnexectomy was performed at 17 weeks of pregnancy. The tumor was diagnosed pathologically as anendocervical-type mucinous cystic tumor of borderline malignancy (Figure 2 and Figure 3). During surgery, no abnormality was observed in the left adnexa, and no finding suggestive of endometriosis was observed in the Douglas fossa or pelvic peritoneum.

After full-term birth with vaginal delivery, a 1-month postpartum checkup with transvaginal ultrasonography revealed a 7 - 8-cm solid left ovarian tumor, which was visualized on magnetic resonance imaging as a 7-cm, irregularly bordered, solid neoplastic lesion in the pelvis, with a signal intensity of low on T1-weighted images and high on T2-weighted images (Figure 4). Hematologic examination showed elevated tumor markers, including 540.9 U/ml CA125, 16.2 U/ml CEA, and $71.7 \mathrm{U} / \mathrm{ml}$ SLX. Given these findings, malignancy was suspected, and exploratory laparotomy was performed 3 months postpartum. The left ovarian tumor invaded the retroperitoneum and reached the anterior aspect of the sacrum while excluding the sigmoid colon to the right. Multiple disseminated lesions of $2 \mathrm{~cm}$ or less in diameter were also found in the upper abdominal peritoneum and peritoneal omentum in the pelvis (Figure 5). Thus, it was decided to remove only the left ovarian tumor and perform
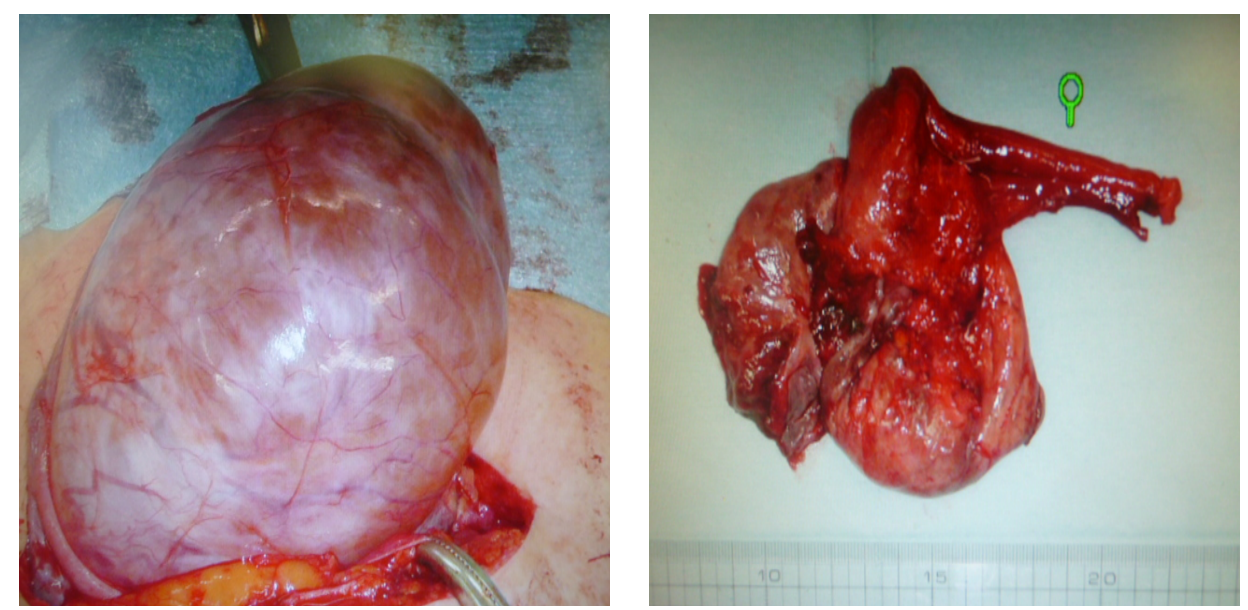

Figure 2. Abdominal right adnexectomy performed at 17 weeks of pregnancy with the second child. The tumor was a multilocular mucinous cyst, the diameter was $15 \mathrm{~cm}$.

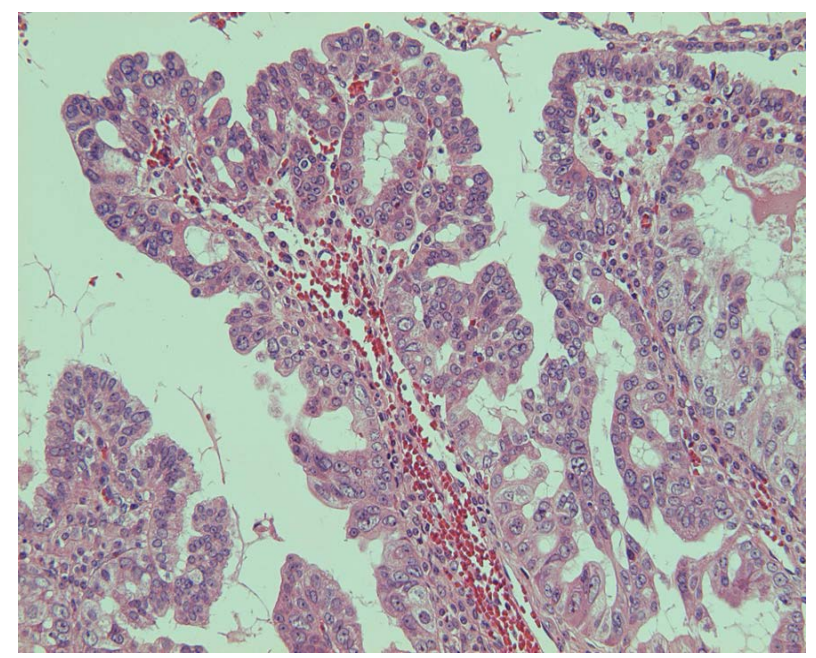

Figure 3. Pathological finding of isolated right adnexa with hematoxylin and eosin stain $\times 40$. It has been shown dyskaryotic cells and mucous retention in cells. Final pathological diagnosis was a mucinous cystic tumor of borderline malignancy. 


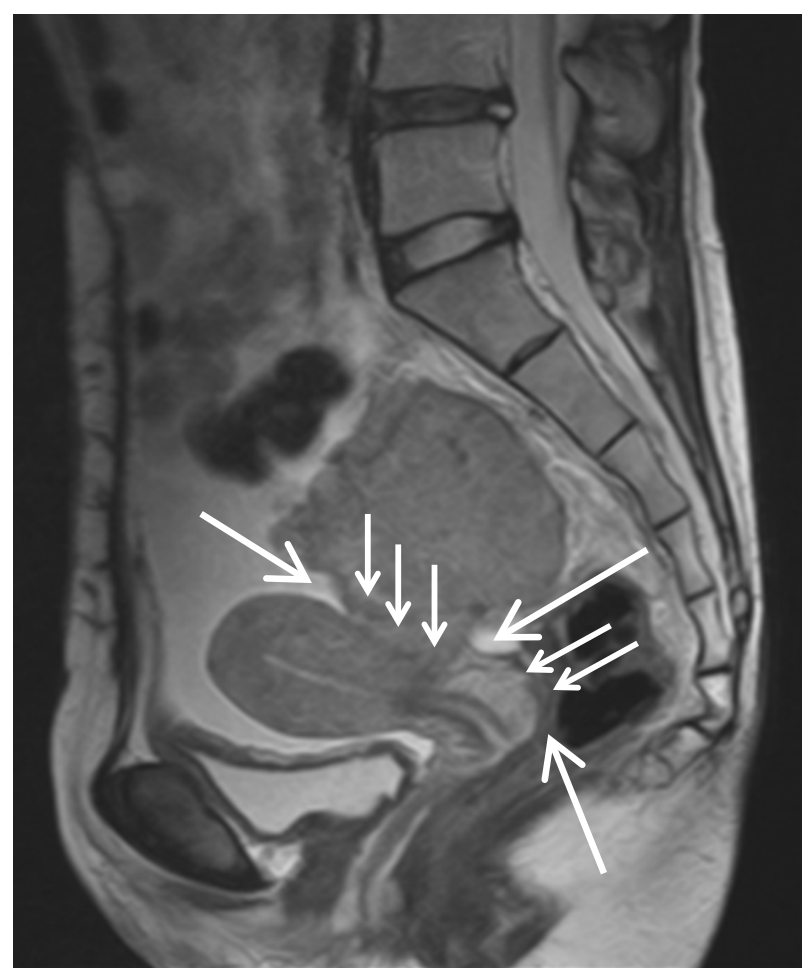

Figure 4. MRI performed 3 months after the delivery of the second child. The left ovarian tumor invaded the retroperitoneum and reached the anterior aspect of the sacrum while excluding the sigmoid colon to the right.

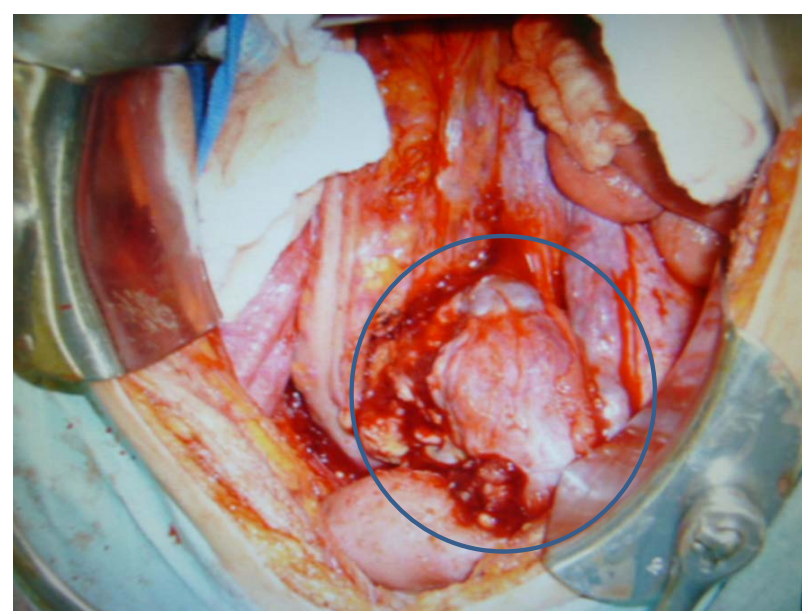

Figure 5. The findings under laparotomy 3 months after the delivery of the second child. The left ovarian tumor was grown retroperitoneal space, compress sigmoid colon, and reached sacral region. Multiple disseminated lesions of $2 \mathrm{~cm}$ or less in diameter were also found in the upper abdominal peritoneum and peritoneal omentum in the pelvis.

biopsy of the omentum. Histopathological diagnosis of the tumor was ovarian carcinoma, endometrioid adenocarcinoma, grade 3, stage IIIb (Figure 6). No clear cell carcinoma component was observed. After surgery, 4 cycles of TC therapy (T: $180 \mathrm{mg} / \mathrm{m}^{2}$; C: AUC5) were given. However, with no response to treatment and deterioration of general condition, the patient died 9 months postpartum. 


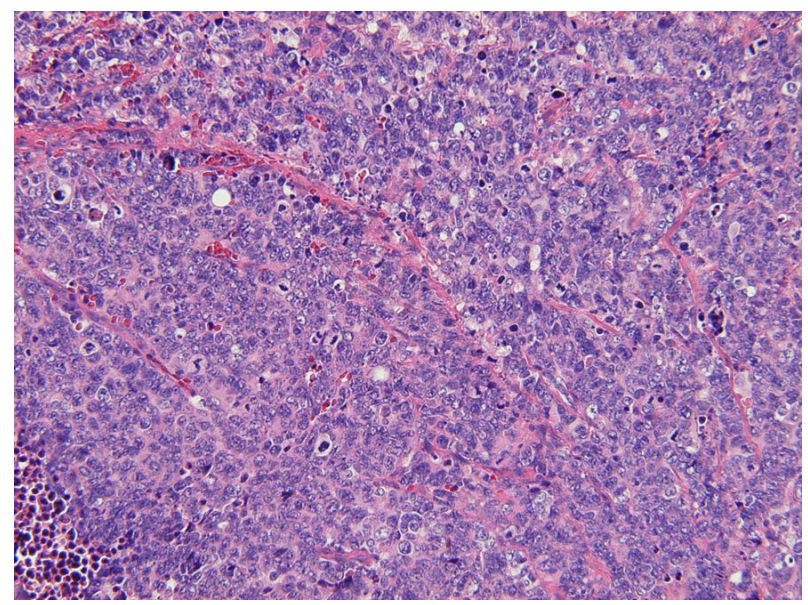

Figure 6. Pathological finding of tumor. The final pathological diagnosis was ovarian carcinoma, endometrioid adenocarcinoma, grade 3 , stage IIIb. There was no finding for clear cell adenocarcinoma $\times 200$.

\section{Discussion}

Two possibilities have been discussed with regard to the relationship between ovarian cancer and fertility treatment. One possibility is that fertility treatment involving ovarian stimulation and controlled ovulation contributes to the development of ovarian cancer. Another possibility is the involvement of patient background factors and predisposing factors of infertility, such as ovulation disorder and endometriosis [5].

To explain the relationship between fertility treatment and ovarian cancer, the following two major theories have been proposed: incessant ovulation therapy with repeated cycles of injury and repair of the ovarian surface epithelium results in gene damage and subsequent cancer development; the high gonadotropin level in gonadotropin therapy mediates the development of ovarian cancer. It has also been suggested that ovulation itself may be a risk factor for the development of ovarian cancer (incessant ovulation therapy) [6]. Whittemore et al. reported that gonadotropin increases the risk of ovarian cancer with an odds ratio of 2.8. Subsequently, Rossing et $a l$. reported that 12 cycles or more of treatment with clomiphene citrate significantly increase the risk of ovarian cancer [7].

Considering that the present patient had received as many as 17 cycles of egg collection, incessant ovulation theory may apply to the present case. However, based on the history of the use of ovulation-inducing agents, gonadotropin therapy cannot be ruled out. On the other hand, other studies have found no correlation between ovarian stimulation and cancer development (Table 2, Table 3), suggesting the need for further follow-up of patients who have undergone fertility treatment.

The prevalence of endometriosis has been reported as 3\% - 10\% among sexually mature women, $25 \%-80 \%$ among infertile women, and $2 \%-5 \%$ among postmenopausal women [8]. Several studies have suggested the involvement of endometriosis - the underlying condition of infertility in the present case - in the development of ovarian cancer. Endometriosis has been shown to correlate significantly with ovarian cancer, with a relative risk of 1.9, compared with 1.2 for all malignancies. The relative risk of ovarian cancer in women with endometriosis has been shown to increase by up to 2.5-fold in those with a follow-up period of at least 10 years [9]. Ovarian endometrial cysts can be found in $17 \%$ - $44 \%$ of all patents with endometriosis [10], and several studies have shown that $0.5 \%-1.0 \%$ of ovarian endometrial cysts develop into ovarian cancer [11]-[13].

Yoshikawa et al. reported a high prevalence of ovarian endometriosis in patients with different histological types of ovarian cancer: approximately $3 \%$ in serous or mucinous adenocarcinoma, $39.2 \%$ in clear cell carcinoma, and $21.2 \%$ in endometrioid adenocarcinoma, with a predominance of early-stage or well-differentiated carcinoma [14]. In the present case, the histological type was endometrioid adenocarcinoma, which is consistent with the findings of previous reports.

A questionnaire survey conducted with members of the Endometriosis Study Group of the Japan Society of Obstetrics and Gynecology Reproductive and Endocrine Committee showed that ovarian cancer can be found in 
Table 2. History of fertility and endometriosis treatment.

\begin{tabular}{|c|c|c|}
\hline Age (years) & Fertility treatment & Endometriosis treatment \\
\hline 24 & Getting married & \\
\hline \multicolumn{3}{|l|}{27} \\
\hline 28 & & 6 cycles of GnRH agonist therapy \\
\hline 29 & $\begin{array}{l}\text { Fertility treatment started } \\
\text { Causes of infertility } \\
\text {-Ovulation disorder }\end{array}$ & \\
\hline 30 & $\begin{array}{l}\text {-Ovulation disorder } \\
\text {-Male factors } \\
+\end{array}$ & $\begin{array}{l}\text { Laparoscopic enucleation of bilateral } \\
\text { ovarian endometrial cysts }\end{array}$ \\
\hline 31 & $\begin{array}{l}\text { Endometriosis } \\
11 \text { sessions of artificial insemination }\end{array}$ & \\
\hline 32 & 17 cycles of egg collection & 6 cycles of GnRH agonist therapy \\
\hline 33 & First child conceived & $\begin{array}{l}\text { Laparoscopic enucleation of bilateral } \\
\text { ovarian endometrial cysts }\end{array}$ \\
\hline \multicolumn{3}{|l|}{34} \\
\hline 37 & Second child conceived naturally & \\
\hline
\end{tabular}

Table 3. Factors contributing to the development of ovarian cancer in infertile women.

\begin{tabular}{|c|c|c|c|c|c|}
\hline Author & Year & $\begin{array}{l}\text { Ovulation/Egg } \\
\text { collection }\end{array}$ & Infertility & Endometriosis & $\begin{array}{c}\text { Ovarian } \\
\text { stimulation }\end{array}$ \\
\hline Fathalla et al. & 1977 & ○ & & & \\
\hline Bames et al. & 2002 & ○ & & & \\
\hline Mundock et al. & 2003 & ० & & & \\
\hline Brinton et al. & 1989 & & ० & & \\
\hline Schidkraut et al. & 1997 & & ० & & \\
\hline Ness et al. & 2002 & & ० & ○ & \\
\hline Craner et al. & 1993 & & ० & & ○ \\
\hline Whittemore et al. & 1992 & & & & $\circ$ \\
\hline Rossing et al. & 1994 & & & & $\circ$ \\
\hline Shushman et al. & 1996 & & & & ○ \\
\hline Venn et al. & 2002 & & & & • \\
\hline
\end{tabular}

$\circ$ Causal relationship $(+)$; $\bullet$ Causal relationship $(-)$.

patients with chocolate ovarian cysts of $\geq 4 \mathrm{~cm}$ in diameter and that its prevalence increases rapidly when cyst diameter exceeds $10 \mathrm{~cm}$. It is thus believed that a chocolate ovarian cyst should be removed if its diameter is 4 cm or more [15].

Here we experienced a case of puerperal ovarian cancer in a patient with an ovarian endometrial cyst and a prior history of long-term fertility treatment. The present case was diagnosed as endometrioid adenocarcinoma, a common type of cancer arising from ovarian endometrial cyst. Although infertility itself has been implicated as a risk factor for the development of ovarian cancer, incessant ovulation therapy or gonadotropin therapy may also be applicable in the present case. Moreover, it remains unclear how pregnancy affects the progression of ovarian cancer. One possibility is that the expanding uterus during pregnancy makes it increasingly difficult to examine the pelvis for early detection of the disease. Further investigations are needed to fully understand the causal relationship between fertility treatment and ovarian cancer development. It is important for physicians to assess history of fertility treatment when they encounter a patient with ovarian cancer. Long-term outcome and 
follow-up of patients who have undergone fertility treatment should also be evaluated in future cases.

\section{References}

[1] Tomao, F., Lo Russo, G., Spinelli, G.P., Stati, V., Prete, A.A., Prinzi, N., Sinjari, M., Vici, P., Papa, A., Chiotti, M.S., Benedetti Panici, P. and Tomao, S. (2014) Fertility Drugs, Reproductive Strategies and Ovarian Cancer Risk. Journal of Ovarian Research, 7, 51. http://dx.doi.org/10.1186/1757-2215-7-51

[2] Rizzuto, I., Behrens, R.F. and Smith, L.A. (2013) Risk of Ovarian Cancer in Women Treated with Ovarian Stimulating Drugs for Infertility. Cochrane Database of Systematic Reviews, 13, CD008215.

[3] Lyttle, B., Bernardi, L. and Pavone, M.E. (2014) Ovarian Cancer in Endometriosis: Clinical and Molecular Aspects. Minerva Ginecologica, 66, 155-164.

[4] Nezhat, F.R., Pejovic, T., Reis, F.M. and Guo, S.W. (2014) The Link between Endometriosis and Ovarian Cancer: Clinical Implications. International Journal of Gynecological Cancer, 24, 623-628. http://dx.doi.org/10.1097/IGC.0000000000000100

[5] Whittemore, A.S., Harris, R. and Itnyre, J. (1992) Characteristics Relating to Ovarian Cancer Risk: Collaborative Analysis of 12 US Case-Control Studies. Invasive Epithelial Ovarian Cancer Group. American Journal of Epidemiology, 136, 1175-1220.

[6] Fathalla, M.F. (1971) Incessant Ovaulation: A Factor in Ovarian Neoplasia? Lancet, 17, 163. http://dx.doi.org/10.1016/S0140-6736(71)92335-X

[7] Rossing, M.A., Daling, J.R., Weiss, N.S., et al. (1994) Ovarian Tumors in a Cohort of Infertile Women. The New England Journal of Medicine, 331, 771-776. http://dx.doi.org/10.1056/NEJM199409223311204

[8] Nezhat, F., Datta, S., Hanson, V., et al. (2008) The Relationship of Endmetriosis and Ovarian Malignancy: A Review. Fertility and Sterility, 90, 1559-1570. http://dx.doi.org/10.1016/j.fertnstert.2008.08.007

[9] Brinton, L.A., Melton, L.J., Malkasian Jr., G.D., et al. (1989) Cancer Risk after Evaluation for Infertility. American Journal of Epidemiology, 129, 712-722.

[10] Chapron, C., Vercellini, P., Barakat, H., et al. (2002) Management of Ovarian Endometriomas. Human Reproduction Update, 8, 591-597. http://dx.doi.org/10.1093/humupd/8.6.591

[11] Brinton, L.A., Sakoda, L.C., Sherman, M.E., et al. (2005) Relationship of Benign Gynecologic Diseases to Subsequent Risk of Ovarian and Uterine Tumors. Cancer Epidemiology, Biomarkers Prevention, 14, 2929-2935. http://dx.doi.org/10.1158/1055-9965.EPI-05-0394

[12] Kobayashi, H. (2005) Chocolate Ovarian Cyst and Malignant Transformation. Acta Obstetricia et Gynecologica Scandinavica Japonzca, 57, N351-N355.

[13] Konishi, I., Ito, K. and Horiuchi, A. (2006) Characteristics and Prognosis of Ovarian Cancer Arising from Endometriosis. Clinical Gynecology and Obstetrics, 60, 134-139.

[14] Yoshikawa, H., Jimbo, H., Okada, S., et al. (2000) Prevalence of Endometriosis in Ovarian Cancer. Gynecologic and Obstetric Investigation, 50, 11-17. http://dx.doi.org/10.1159/000052873

[15] Kobayashi, H. (2005) Adenomyosis/Endometriosis: Chocolate Ovarian Cyst and Malignant Transformation. Acta Obstetricia et Gynecologica Scandinavica Japonzca, 57, 351-355. 